\title{
SYNTHESIS AND CHARACTERIZATION OF PVA CAPPED CdS
}

\section{NANOPARTICLES}

\section{K. Vishwakarma ${ }^{1}$, T. Nanhoriya ${ }^{1}$, D. Ahirwar ${ }^{2}$, S. Khade ${ }^{3}$, S. Tiwari ${ }^{4}$}

${ }^{1}$ Dept. of Nanotechnology, Gyan Ganga College of Technology, Jabalpur,

E-mail: triptinanhoriya@gmail.com

\begin{abstract}
CdS is an important II-VI semiconductor and has been highlighted as an efficient emitter because of its high quantum yield and direct band gap in the visible range. In the present work, PVA capped CdS nanoparticles have been synthesized using Simple Chemical route. This paper presents the study of the structural and optical properties of PVA capped cadmium sulphide nanoparticles. XRD pattern showed the hexagonal phase o\f CdS nanoparticles. The band gap of the nano crystalline material is determined from the UV spectrograph. The calculated grain size is found to be $3.62 \mathrm{~nm}$.
\end{abstract}

Keywords: Nanocrystals, PVA, SEM, XRD, UV-Vis spectroscopy, FTIR.

\section{INTRODUCTION}

The semiconductor nanoparticles exhibit structural, optical, electronic, Luminescence and photo-conducting properties [1] very different from their bulk properties. It is also attractive because of their possible application in solar cell, photo-detector, Laser, LED, high density magnetic information storage and many others in semi conducting industries [2]. Quantum dots of II-VI semiconductors have d particular attention, because they are easy to synthesize in the size range required for quantum confinement. So II-VI semiconductors with dimension in the nanometer range have generated considerable interest for researcher and scientific community. CdS nanoparticles exhibit size dependent properties it has a band gap energy $\mathrm{E}_{\mathrm{g}}$ of $2.42 \mathrm{eV}$ at room temperature and pressure. Since CdS has wide band gap, it is used as window material for heterojunction solar cells to avoid the recombination of photo-generated carriers which improves the solar cell efficiency [3]. 
Semiconductor nanoparticles are themselves highly unstable, and in the absence of capping agent, they agglomerate very rapidly. For this reason, bonding of capping agents to nanoparticles is necessary to provide chemical passivation and also to improve the surface state which has substantial influence on the optical and the electronic properties of nanoparticles. Polymer capped nanoparticles composites have been increasingly studied [4].

\section{SYNTHESIS: MATERIALS AND METHODS}

Cadmium Sulphide nanoparticles were grown by Chemical Bath method using PVA as a capping agent. All the chemical reagents used were of analytical grade. For the undoped CdS nanoparticles, the solution of $\mathrm{CdSO}_{4}(0.5 \mathrm{M})$ was prepared by dissolving $\mathrm{CdSO}_{4}$ in DI water. The matrix solution was prepared by adding the $\mathrm{CdSO}_{4}$ solution to an aqueous solution $(2 \%)$ of PVA with constant stirring at room temperature to form a well dispersed PVA capped $\mathrm{Cd}^{2+}$ ion solution which is a transparent liquid indicating the complete dissolution of $\mathrm{CdSO}_{4}$. The $\mathrm{pH}$ of the solution was maintained at around 8 by slowly adding Ammonium Buffer Solution drop by drop to the above matrix solution to form the metallic complex. The thiourea as $\mathrm{S}^{2--}$ ion source was then added to the above metallic complex solution to form colloidal solution of CdS nanoparticles.

\section{CHARACTERIZATION}

The prepared sample was taken for XRD study and UV studies. The structure of the sample is determined by X-ray diffraction measurements have been performed by using Xpert PANalytical instrument operating at $40 \mathrm{kV}$ and current of $30 \mathrm{~mA}$ with CuKa radiation. FTIR measurements are recorded using FTIR-8400s SHIMADZH (IR solution). UV-Visible spectrum of the nanoparticles is recorded using PERKIN ELMER UV-Vis spectrophotometer. 


\section{RESULTS AND DISCUSSION}

\section{SEM ANALYSIS:}

Scanning electron microscope (SEM) study: SEM is a type of electron microscope that images the sample surface by scanning it with a highenergy beam of electrons in a raster scan pattern. Here, it is used to characterize the morphology of the nanoparticles.

Fig. 1 shows the SEM micrographs of the CdS nanoparticles. The grain size was measured from SEM photograph by keeping the photograph under traveling microscope having high accuracy. It was observed that the grain size have nano-metric dimensions ranging from $10.26 \mathrm{~nm}$ to $22.12 \mathrm{~nm}$.

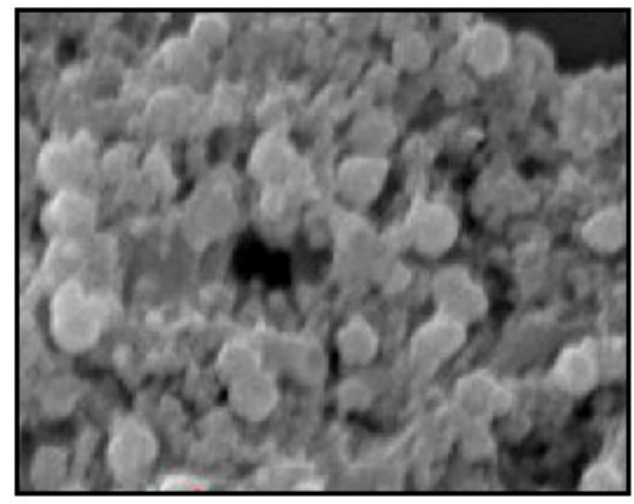

Figure 1: SEM image of PVA capped CdS sample.

\section{UV-Visible STUDY:}

Considering the band gap energy of bulk CdS as $2.42 \mathrm{eV}$, large blue shift is observed in both CdS and Cu doped CdS samples as shown in figure.. This indicates a clear quantum confinement in PVA capped CdS nanostructures. C.M. Janet et al reported that when the size of CdS nanocrystal becomes smaller than the exciton radius a remarkable quantum size effect leads to a size dependent increase in the band gap and a blue shift in the absorption onset [5]. 
Large blue shift is observed in CdS sample with respect to bulk CdS. This indicates a clear quantum confinement in PVA capped CdS nanostructures. C.M. Janet et al also reported that, the size of CdS nanocrystal becomes smaller than the exciton radius a remarkable quantum size effect leads to a size dependent increase in the band gap and a blue shift in the absorption onset [6].

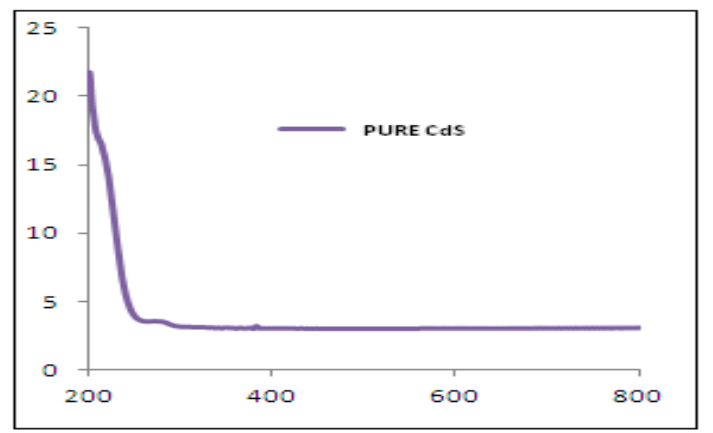

\section{Figure 2. Absorption spectra of PVA capped CdS nanoparticles XRD STUDY:}

The structure of the prepared sample was investigated by $\mathrm{X}$-Ray Diffractometer technique. The XRD pattern of the sample is shown in fig. The particle size was calculated by using Debye- Scherrer formula.

$$
\mathrm{D}=\mathrm{k} \lambda / \beta \cos \theta
$$

Where, $\mathrm{k}$ is a dimensionless constant, $\lambda$ is the wavelength of $\mathrm{X}$-Ray used, $\beta=$ Full width at Half maxima (FWHM) of the diffraction peak and $\theta$ is the diffraction angle for the $(h, k, l)$ plane Bragg's angle. XRD shows hexagonal CdS nanoparticles with size in a few nanometers range. Significant peaks were obtained at $2 \theta$ angles $26^{\circ}, 29^{\circ}, 32^{\circ}, 36^{\circ}, 44^{\circ}, 51.5^{\circ}, 61.5^{\circ}$ corresponds to the reflections at (002), (101), (200),102), (110), (112), (202) planes. 


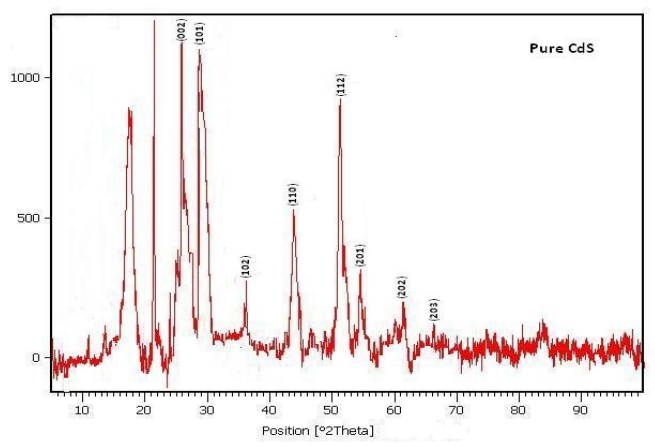

Figure 3: XRD spectra for PVA capped CdS sample.

\section{FTIR STUDY:}

In FTIR spectrum, the peak at $3431 \mathrm{~cm}^{-1}$ is assigned to $\mathrm{O}-\mathrm{H}$ stretching of absorbed water on the surface of the sample and the peak at $1438 \mathrm{~cm}^{-1}$ is attributed to bending vibrations of Poly Vinyl Alcohol used in the process. The $\mathrm{C}-\mathrm{O}$ stretching vibration of absorbed PVA molecule gives its intense peak at $1025 \mathrm{~cm}^{-1}$. In addition to surface coverage of CdS by PVA, presence of trace amount of template ligand namely PVA is also evident, its ring $\mathrm{C}-\mathrm{H}$ vibration occurs at about $3074 \mathrm{~cm}^{-1}$ which is a very weak peak. The FT-IR spectra for PVA capped CdS sample is shown in figure 4.

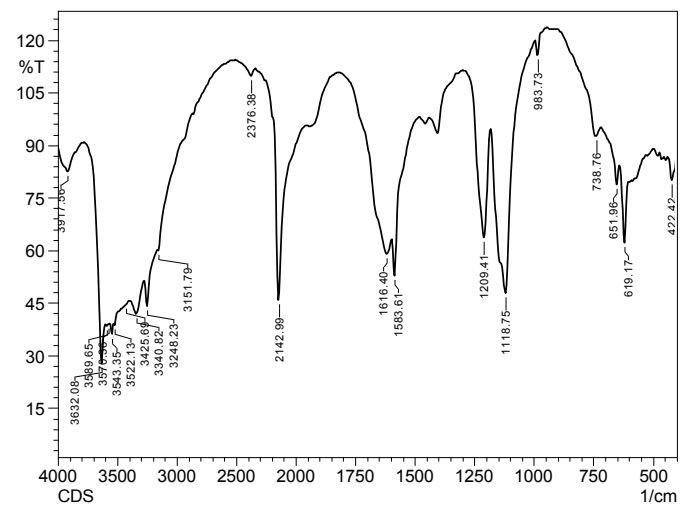

Figure 4: FTIR spectra for PVA capped CdS sample. 


\section{CONCLUSIONS}

CdS nanoparticles have been synthesized in the PVA matrix through simple chemical route. X-ray diffraction confirms the hexagonal structure. UV-visible absorption showed a blue shift indicating quantum confinement of the particle. The obtained results can be useful for the started point of photonic device fabrication.

\section{REFERENCES}

Charles Kittel, (1995) Introduction to Solid State Physics, 7.

S. A. Al Kuhaimi, (1998) Sol. Energy Mater. Sol. Cells 52, 69 [DOI: 10.1016/S0927-0248(97)002729].

H.S. Nalwa; (2000) Handbook of nanostructured materials and nanotechnology, Vol-1, Academic Press.

Qi L., Colfen H., Antonietti M., (2001) Synthesis and Characterization of CdS nanoparticles Stabilized by Double-Hydrophilic Block Copolymer, Nano Lett., 1, 61-65.

S.Salimian and S.Farjami Shayesteh, (2010) Luminescence Properties of CdS Nanoparticles under Various Synthesis Conditions, Vol. 118, pp.4.

C M Janet and R P Viswanath, (2006) Large Scale synthesis of CdS nanorods and its utilization in photo catalytic H2 production technology, Nanotechnology 17, 5271-5277. 